\title{
Proposals for the set of pairwise comparisons
}

\begin{abstract}
Pairwise comparison matrix is a fundamental concept of the AHP. In this paper we provide recommendations for filling in patterns of incomplete pairwise comparison matrices, when we assume that the set of comparisons can be chosen and it is designed completely before the decision making process, without any further prior information. Our recommendations heavily rely on the graph representation of the incomplete pairwise comparison matrices, in fact, the suggested designs are represented by (quasi-)regular graphs with minimal diameter. One major contribution of our research is a list of proposed graphs for different parameter sets, where it is important to emphasize that the diameter of the graph has not been studied in the literature as a crucial property before. On the other hand, we validate the results with the help of extended numerical simulations, which show that these filling in patterns provide smaller errors compared to other well-known designs. Both theorists and practitioners can utilize the results, provided in several formats in our research: graph, adjacency matrix, list of edges. Also, a lot of other models based on pairwise comparisons can take advantage of our findings.
\end{abstract}

Keywords: pairwise comparison, incomplete pairwise comparison matrix, graph, regular graph, diameter

\section{Introduction}

The concept of pairwise comparison matrices is one of the most important and fundamental ones in the Analytic Hierarchy Process (Saaty, 1980). In the last decades pairwise comparisons have become popular in the theory and practice of preference modelling and quantification, while dealing with incomplete data also gets more and more attention in the literature. We focus on decision problems where the set of pairwise comparisons can be chosen, i.e., it is not given a priori. We also assume that we do not have any further prior information about the items to be compared, and the set of comparisons is designed completely before the decision making process.

The objective of this paper is to provide recommendations for filling patterns of incomplete pairwise comparison matrices based on their graph representation. From a mathematical point of view, the aim of our research is to find graphs, among regular and quasi-regular ones, with minimal diameter.

Regularity means that each item is compared to others for the same number of times, resulting in a kind of symmetry. A graph on an odd number of vertices is called quasiregular, if the degree of every vertex is the same odd number, except for one vertex whose degree is larger by one.

If there is a pair of items such that their shortest connecting path is very long, the comparison between these two elements is possibly biased by all of the errors of the intermediate comparisons, so the calculated results can be misleading. Such an example was found in Tekile (2017), which particularly inspired our research. If the diameter (the 
longest shortest path) of the graph of comparisons is as low as possible, we can avoid or at least decrease the abovementioned cumulated errors leading to a better estimation of the original preferences.

\section{Literature Review}

Kulakowski et al. (2019) recently created an incompleteness index based on the number of missing pairwise comparisons and their arrangements, where regularity is of special importance. There are already known particular recommended structures for filling in incomplete pairwise comparison matrices, moreover few of those also include some kind of (usually a stronger type of) regularity as well (Wang and Takahashi, 1998). However those are not as general as our approach, and the diameter of the representing graph has not been examined in the literature so far, thus we would like to fill in this gap.

Our work also has a strong connection with the degree/diameter problem that is well known in the mathematical literature, for an extensive summary see for instance Miller and Širán (2013).

\section{Hypotheses/Objectives}

In our research we would like to provide a list of graphs which shows the patterns of the comparisons that have to be made in case of different number of items to be compared (denoted by $\mathrm{n}$ ), different level of (quasi-)regularity and different minimal diameters (denoted by d). Furthermore it is a key step to validate our hypothesis that our recommended filling in patterns can perform better compared to other designs based on different error measurements.

\section{Research Design/Methodology}

In order to provide the list of graphs, in smaller cases we used different computational methods to generate all the possible regular graphs and select those with the smallest diameter. For larger instances we used several graph construction techniques as generating all the possible regular graphs became almost impossible in these cases. Creating such a list of graphs, or only finding one graph for a given parameter set turned out to be computationally really challenging.

On the other hand, we use several examples and extended simulation experiments to compare our recommendations with other filling in pattern designs with the same number of comparisons. We evaluate the results based on different types of distance measures and several types of priority vector calculation techniques.

\section{Data/Model Analysis}

A small sample from the list of (quasi-)regular graphs with minimal diameter:

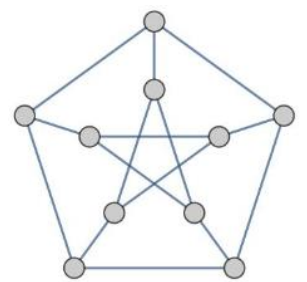

Petersen graph (3-regular, $n=10, d=2$ )

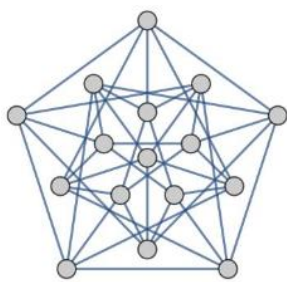

Clebsch graph (5-regular, $n=16, d=2$ )

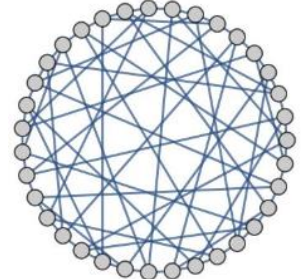

Odd-4 graph (4-regular, $n=35, d=3$ )

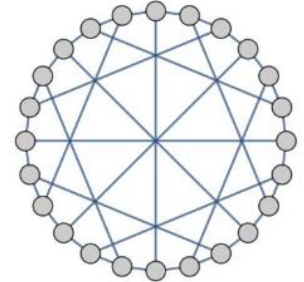

McGee graph (3-regular, $n=24, d=4$ )

International Symposium on the 


\section{Limitations}

Despite our simulation based approach can provide some evidence on the validity of our recommendations, it would be indeed nice to structure our results in a theorem, however we could not solve this problem yet. Moreover, the computational demands of the problems are obviously a limitation considering the size of the graphs in our list.

\section{Conclusions}

We provided a list of graphs showing our recommendation for filling in patterns of incomplete pairwise comparison matrices, namely (quasi-)regular graphs with minimal diameter. We discussed regularity more generally compared to many studies in the literature, and we are also the first to consider the diameter of the representing graph as an important property. The validation of our recommendations was successful with the help of numerical simulations.

The target of our future research can be the determination of even larger graphs. Also, when there are several graphs with the needed properties, we can reduce their number based on some chosen centrality measures, for instance, instead of the diameter, we can concentrate on the average path length that could lead to an indeed interesting study. In case of the current research, both theorists and practitioners can utilize the results, provided in several formats: graph, adjacency matrix, list of edges. Although our conclusions have been presented within the framework of pairwise comparison matrices, they are applicable in a wider range. A lot of other models based on pairwise comparisons can take advantage of our findings.

\section{Key References}

Kulakowski, K., Szybowski, J., and Prusak, A. (2019). Towards quantification of incompleteness in the pairwise comparisons methods. International Journal of Approximate Reasoning, 115, 221-234.

Miller, M. and Širáň, J. (2013). Moore graphs and beyond: A survey of the degree/diameter problem. Electronic Journal of Combinatorics, 20(2), 1-92.

Saaty, T. L. (1980). The Analytic Hierarchy Process. McGraw-Hill, New York.

Tekile, H. A. (2017). Incomplete pairwise comparison matrices in multi-criteria decision making and ranking. Master's thesis, Central European University.

https://mathematics.ceu.edu/sites/mathematics.ceu.hu/files/attachment/basicpage/29/thesi shailemariam.pdf

Wang, K. and Takahashi, I. (1998). How to select paired comparisons in AHP of incomplete information - strongly regular graph design. Journal of the Operations Research Society of Japan, 41(2), 311-328. 